\title{
Fusion of Near Infrared Face and Iris Biometrics*
}

\author{
Zhijian Zhang ${ }^{1}$, Rui Wang ${ }^{2}$, Ke Pan $^{1}$, Stan Z. Li ${ }^{2}$, and Peiren Zhang ${ }^{1}$ \\ ${ }^{1}$ University of Science and Technology of China \\ Hefei 230026, China \\ ${ }^{2}$ Center for Biometrics and Security Research \& \\ National Laboratory of Pattern Recognition \\ Institute of Automation, Chinese Academy of Sciences, Beijing 100080, China \\ http://www.cbsr.ia.ac.cn
}

\begin{abstract}
In this paper, we present a method for fusing face and iris biometrics using single near infrared (NIR) image. Fusion of NIR face and iris modalities is a natural way of doing multi-model biometrics because they can be acquired in a single image. An NIR face image is taken using a high resolution NIR camera. Face and iris are segmented from the same NIR image. Face and iris features are then extracted from the segmented parts. Matching of face and iris is done using the respective features. The matching scores are fused using various rules. Experiments give promising results.
\end{abstract}

Keywords: NIR imaging, face, iris, multimodal biometrics, score fusion.

\section{Introduction}

Unimodal biometric systems perform person recognition based on a single source of biometric information. Face and iris recognition systems are of best choices. Face recognition is non-invasive and friendly; also, it is easy to get face images of good quality. Iris recognition is one of the most accurate biometrics. Unfortunately, both of them are often affected by a number of practical issues. The accuracy of face recognition is affected by illumination, pose, shelter and facial expression. In the case of iris recognition, the user must be cooperative to get iris images of good quality, because iris images must meet critical quality requirement. This may annoy the users.

Fusion of face and iris could overcome some of the limitations mentioned above and bring the following advantages [1. 1) The total error rate (the false accept rate and the false reject rate) is known to go down 2. 2) It reduces spoof attacks on the biometric system. Fusion of face of iris is a good method for liveness detection because of the difficulty in making fake iris images. 3) The population coverage of a multimodal biometric system is larger than that of

\footnotetext{
* The work presented in this paper was performed at CBSR during Zhijian Zhang and Ke Pan's visiting CBSR as visiting students.
} 
a standalone biometric system. The use of the face recognizer can allow people with imperfect iris images to enroll, reducing the enrollment failure rate. Wang [2] used two different strategies for fusing iris and face classifiers. The first strategy was to compute either an unweighted or weighted sum and compare the result to a threshold. The second strategy was to treat the matching distances of face and iris classifiers as a two-dimensional feature vector and use a classifier to classify the vector to genuine or impostor. Byungjun Son [3] extracted features of face and iris by wavelet, and fused face and iris at feature level using reduced joint feature vector.

Two disadvantages exist in the conventional way of face+iris fusion. Firstly, face images are captured in the visible light (VL) spectrum. Lighting conditions influence the appearance of a face 4, and hence the accuracy of the face modality. Secondly, face images and iris images are captured using different imaging devices.

In this paper, we present a new method for fusion of face and iris biometrics, by taking advantages of the recently developed near infrared (NIR) face imaging and recognition [5]. NIR face and iris data are acquired using single high resolution camera with active frontal NIR lighting. This is not only a natural way for face and iris multimodal biometrics, because both modalities need active NIR modality, but also brings convenience to the user.

Face and iris regions are segmented from the same image, with sufficient details because of the high resolution. Face and iris biometric features are then extracted. They are matched against face and iris templates using the respective features. The matching scores are fused to give the final result. Experiments give promising results, with various fusion rules compared.

The rest of the paper is organized as follows: Section 2 introduces the image acquisition method and the algorithm structure for fusing face+iris biometrics using a single NIR face image. The database and experiments are given in section 3 .

\section{Face+Iris Multimodal Fusion}

\subsection{NIR Image Acquisition}

The image resolution should be high enough to capture sufficient iris details. A typical iris image size is $320 \times 280$ pixels and the area of a face is 100 times larger than that of iris. So the resolution of the resulting image should be more than 9 megapixel pixels $(320 \times 280 \times 100=8,960,000)$. Therefore, we choose to use a 10 megapixel CCD digital camera with up to $3648 \times 2736$ pixels. The camera is about $60-80 \mathrm{~cm}$ away from the subject.

To overcome influence of ambient lighting on face and to get iris patterns, active NIR LED lights of $850 \mathrm{~nm}$, mounted around the camera lens, are used to provide frontal lighting. We use a band pass optical filter on the camera lens to cut off visible light while allowing NIR light to pass. Figure 1 shows some examples of face images and segmented iris parts. 

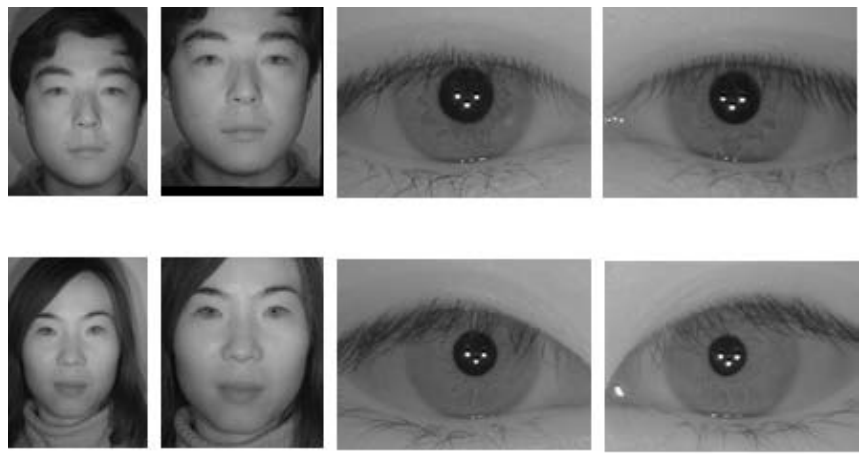

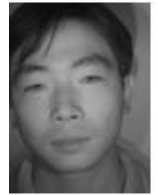

(a)

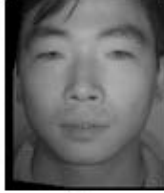

(b)

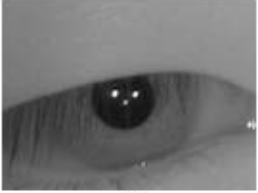

(c)

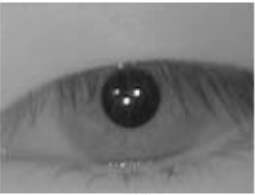

(d)

Fig. 1. High resolution NIR face images and corresponding face and iris images. (a) High resolution NIR face images. (b) NIR face image segmented from (a). (c) Left iris segmented from (a). (d) Right iris segmented from (a).

\subsection{Face and Iris Recognition}

We use the PCA [6] for face recognition. Suppose $I_{1}, I_{2}, \ldots, I_{n}$ as a training set, its average face is defined by:

$$
A=\frac{1}{S} \sum_{i=1}^{S} I_{i}
$$

The margin between a face image and the average face is $Y_{i}=I_{i}-A$. Covariance matrix $C$ is defined by:

$$
C=\frac{1}{S} \sum_{i=1}^{S} Y_{i} \cdot Y_{i}^{T}
$$

Let $E^{T}$ be $M$ maximum eigenvectors of $C$. Test image $I_{\text {test }}$ projects to the face space as the algorithm:

$$
W_{\text {test }}=E^{T} \cdot\left(I_{\text {test }}-A\right)
$$

We use the well-known iris recognition algorithm of Daugman [7. It comprises 4 steps. 1) It is necessary to localize precisely the inner and outer boundaries of the iris, and to detect and exclude eyelids if they intrude. 2) The portion of the image corresponding to the iris is translated to a normalized form, so that possible dilation of the pupil does not affect the system. 3) The feature extraction process is completed by the use of 2D Gabor wavelets to perform 
a multi-scale analysis of the iris. The information about local phase, coded with two bits corresponding to the signs of the real and imaginary parts, is obtained. The result is a 256-byte IrisCode. 4) Similarity scores are obtained by computing a Hamming Distance.

\subsection{Score Level Fusion}

There are two approaches for fusion at score level fusion. The first one is to formulate it as a classification problem. The second one is to treat it as a combination problem. The individual matching scores are combined to generate a single scalar score which is then compared to a threshold to make the final decision. To ensure a meaningful combination of scores, the scores must be transformed to a common domain. Normalization methods and combination approaches have been proposed in literatures 8,9.

Min-Max is a well-known normalization method:

$$
n=\frac{s-\min (S)}{\max (S)-\min (S)}
$$

Where, $S$ is the score set composed of all scores for a matcher, $s \in S, n$ is the normalized score, $\max (S)$ and $\min (S)$ specify the end points of $S$.

Two score level fusion methods, sum-rule (Eq. 5) and product rule (Eq. 6), are used in the work. Suppose $f_{i}$ as the fused score for user $i$,

$$
\begin{aligned}
& f_{i}=\sum_{m=1}^{M} n_{i}^{m} \\
& f_{i}=\prod_{m=1}^{M} n_{i}^{m}
\end{aligned}
$$

Where, $n_{i}^{m}$ is the normalized score for matcher $m$ applied to user $i, m=$ $1,2, \ldots, M, M$ is the number of matchers, $i=1,2, \ldots, I, I$ is the number of individuals.

\subsection{Summary of Algorithm}

Figure 2 summarizes the structure of the algorithms of NIR face+iris biometric fusion using a single high resolution NIR face image:

1. Image Capturing: a high resolution NIR face image;

2. Face and eye localization: using a face and eye detection algorithm [10;

3. Segmenting irises from the face;

4. Normalizing the face and irises in given frames;

5. Extracting facial features using PCA [6];

6. Extracting iris features using Gabor [7;

7. Performing face and iris matching against respective templates using respective features; 


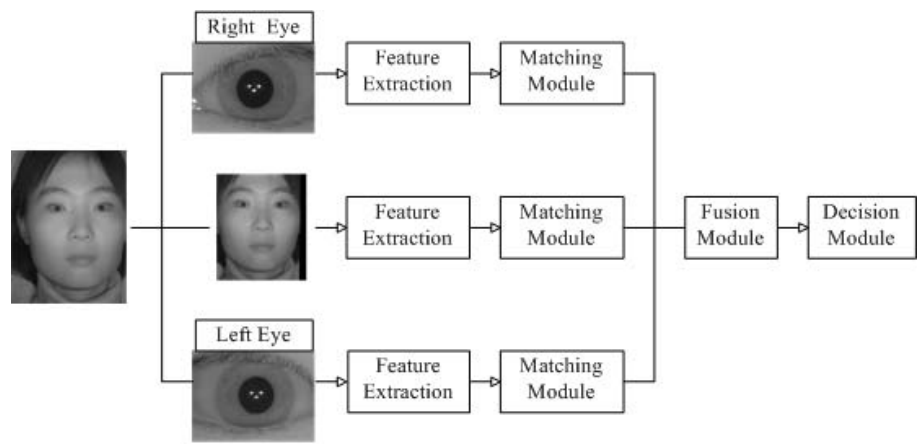

Fig. 2. Algorithm structure for fusing face+iris biometrics using a single NIR face image

8. Fusing the matching scores [89];

9. Comparing the fused score to a threshold to make the final decision.

The final matching scores of face and iris are normalized to $[0,1]$ range using MinMax normalization. A single scalar score is obtained using sum rule and product rule. The NIR face+Iris fusion may be done with different combinations: 1) fusion of face and left iris, 2) fusion of face and right iris, 3) fusion of face, left iris and right iris and 4) fusion of left iris and right iris.

\section{$3 \quad$ Experiments}

\subsection{Database}

A NIR face+iris database is built which contains 930 high resolution $(2736 \times 3648$ pixels) NIR images. It includes 112 subjects of 55 females and 57 males, aged from 17 to 35, with 10 images for 76 subjects and 5 images for other 34 subjects. Some examples were shown in Figure 1.

The images in the database are divided into three parts, training set, test set 1 (a close set) and test set 2 (an open set). The training set includes 380 images (76 subjects, 5 images for each subject). The test set 1 includes 380 images (76 subjects, 5 images for each subject) with the same subjects as those of the training set. The test set 2 includes 170 images (34 subjects, 5 images for each subject) with totally different subjects from those of the training set. Test set 2 has more poor quality iris images than test set 1 .

\subsection{Results}

Face and iris matching scores are obtained using the PCA and Gabor based methods. We use Min-Max to normalize the matching scores output from face/ iris verification. We use product rule and sum rule to combine the normalized scores. 
Table 1. GAR(\%) $(F A R=0.001$, Min-Max Normalization $)$

\begin{tabular}{|l|l|l|l|l|}
\hline & $\begin{array}{l}\text { Test Set 1 } \\
\text { Sum Rule }\end{array}$ & $\begin{array}{l}\text { Test Set 1 } \\
\text { Product Rule }\end{array}$ & $\begin{array}{l}\text { Test Set 2 } \\
\text { Sum Rule }\end{array}$ & $\begin{array}{l}\text { Test Set 2 } \\
\text { Product Rule }\end{array}$ \\
\hline Face & 97.35 & 97.35 & 83.31 & 83.31 \\
\hline Left & 95.42 & 95.42 & 88.0 & 88.0 \\
\hline Right & 95.0 & 95.0 & 86.33 & 86.33 \\
\hline Face+Left & 99.36 & 99.39 & 97.24 & 97.34 \\
\hline Face+Right & 98.65 & 99.49 & 92.63 & 94.17 \\
\hline Left+Right & 97.64 & 98.0 & 93.29 & 93.80 \\
\hline Face+Left+Right & 99.75 & 99.74 & 98.12 & 97.81 \\
\hline
\end{tabular}

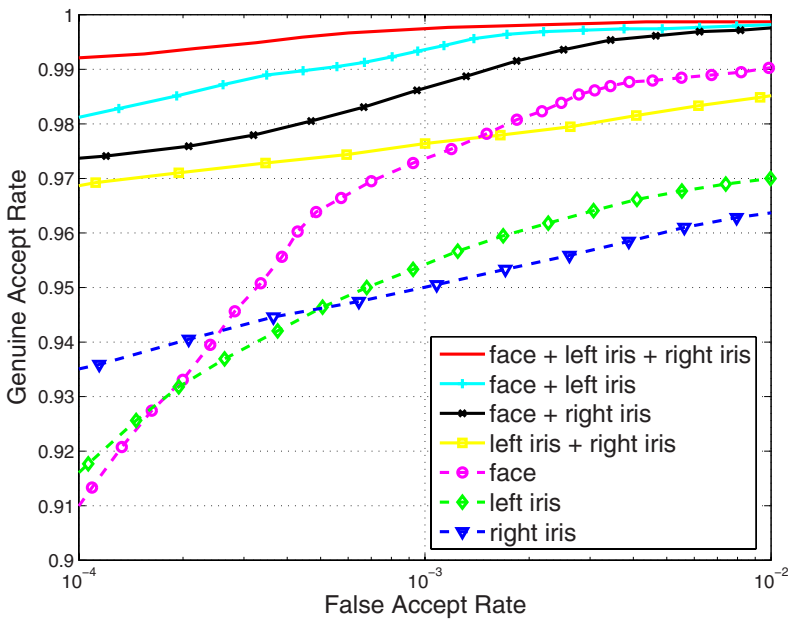

(a) Min-Max normalization and sum rule

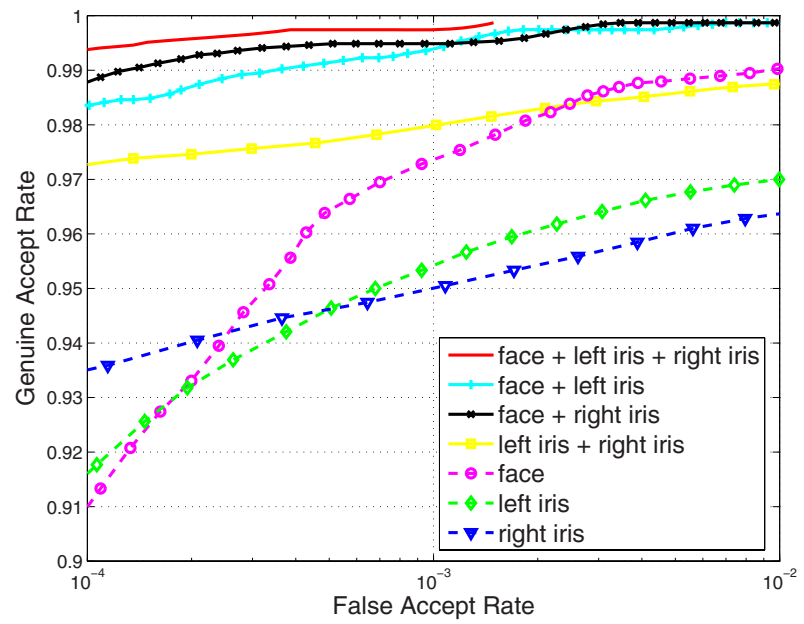

(b) Min-Max normalization and product rule

Fig. 3. ROC Curves for score level fusion of face and iris on test set 1 (close set) 
Table 2. $\operatorname{GAR}(\%)(F A R=0.0001$, Min-Max Normalization $)$

\begin{tabular}{|l|l|l|l|l|}
\hline & $\begin{array}{l}\text { Test Set 1 } \\
\text { Sum Rule }\end{array}$ & $\begin{array}{l}\text { Test Set 1 } \\
\text { Product Rule }\end{array}$ & $\begin{array}{l}\text { Test Set 2 } \\
\text { Sum Rule }\end{array}$ & $\begin{array}{l}\text { Test Set 2 } \\
\text { Product Rule }\end{array}$ \\
\hline Face & 91.0 & 91.0 & 71.73 & 71.73 \\
\hline Left & 91.60 & 91.60 & 84.47 & 84.47 \\
\hline Right & 93.51 & 93.51 & 77.36 & 77.36 \\
\hline Face+Left & 98.12 & 98.32 & 94.55 & 95.0 \\
\hline Face+Right & 97.37 & 98.78 & 90.77 & 90.0 \\
\hline Left+Right & 96.86 & 97.27 & 90.65 & 90.75 \\
\hline Face+Left+Right & 99.21 & 99.38 & 95.30 & 95.88 \\
\hline
\end{tabular}

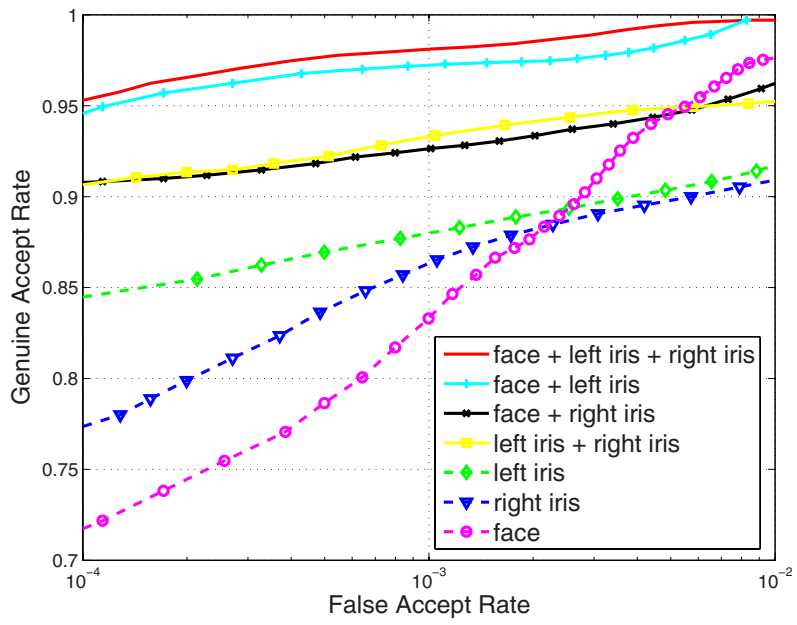

(a) Min-Max normalization and sum rule

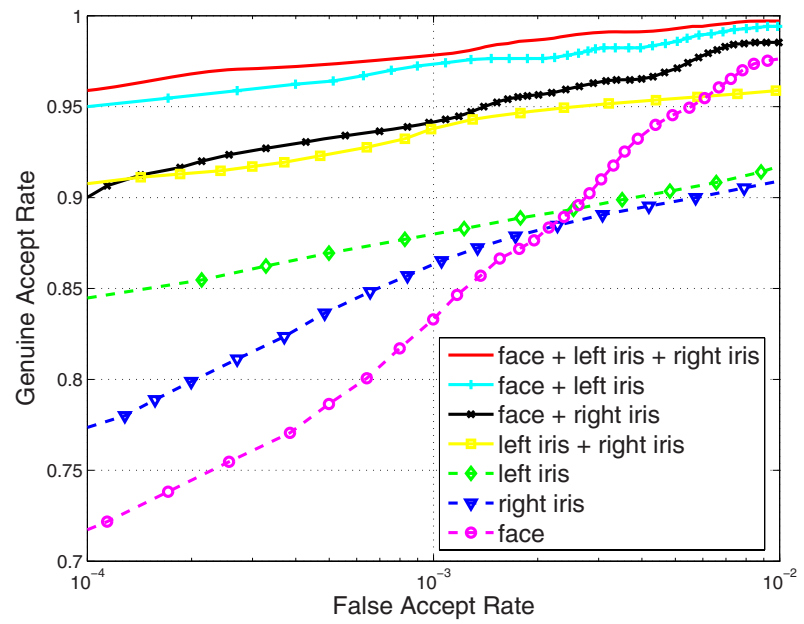

(b) Min-Max normalization and product rule

Fig. 4. ROC Curves for score level fusion of face and iris on test set 2 (open set) 
Table 1 shows the genuine acceptance rate (GAR) when false acceptance rate (FAR) is at 0.001. Table 2 shows the GAR when FAR is at 0.0001. Figure 3 shows the ROC curves for score level fusion of face and iris obtained on test set 1. Figure 4 shows the ROC curves for score level fusion of face and iris obtained on test set 2 .

\subsection{Conclusions}

Two conclusions may be drawn based on Figures 3 and 4 and Tables 1 and 2

1) Fusion of NIR face and irises can improve the accuracy. In case of fusion of face and two irises, the genuine accept rate (GAR) increases from $97.35 \%$ (face biometric) to $99.75 \%$ on a close set and from $83.31 \%$ (face biometric) to $98.12 \%$ on an open set when false accept rate (FAR) is at 0.001 . Fusion of multiple biometrics has a higher accuracy than fusion of multiple units of the same biometric.

2) Multimodal biometrics is a way to reduce the quality requirement of iris image. Some samples, such as the third image in Figure1(c), fail the standalone iris classifier, but correctly recognized by the multimodal biometric system.

\section{Summary and Future Work}

In this work, we present a method for fusing NIR face and iris biometrics using single high resolution NIR face image. The image is taken with frontal active NIR illumination. This provides frontal NIR lighting for face image and iris pattern acquisition at the same time. Preliminary experiments shows that fusion of NIR face and iris biometrics can improve the accuracy.

We will be considering to integrate the two modalities in a better way to take advantages of both: (1) that NIR face biometric has a good accuracy, is fast and does not so stringent user cooperation, and (2) that iris biometric could have a higher accuracy, if the imaging is done properly, due to better separability of iris patterns. A careful design of integration of the NIR face and iris may lead to a system which has a high accuracy, and is fast, and easy to use.

\section{Acknowledgments}

This work was supported by the following funding resources: National Natural Science Foundation Project \#60518002, National Science and Technology Supporting Platform Project \#2006BAK08B06, National 863 Program Projects \#2006AA01Z192 and \#2006AA01Z193, Chinese Academy of Sciences 100 people project, and the AuthenMetric Collaboration Foundation.

\section{References}

1. Hong, L., Jain, A., Pankanti, S.: Can multibiometrics improve performance? In: Proceedings AutoID'99, pp. 947-950 (1999)

2. Wang, Y., Tan, T., Jain, A.K.: Combining face and iris biometrics for identity verification. In: Kittler, J., Nixon, M.S. (eds.) AVBPA 2003. LNCS, vol. 2688, pp. 805-813. Springer, Heidelberg (2003) 
3. Son, B., Lee, Y.: Biometric authentication system using reduced joint feature vector of iris and face. In: Kanade, T., Jain, A., Ratha, N.K. (eds.) AVBPA 2005. LNCS, vol. 3546. Springer, Heidelberg (2005)

4. Moses, Y., Adini, Y., Ullman, S.: Face recognition: The problem of compensating for changes in illumination direction. In: Proceedings of the European Conference on Computer Vision, vol. A, pp. 286-296 (1994)

5. Li, S.Z., Chu, R., Liao, S., Zhang, L.: Illumination invariant face recognition using near-infrared images. IEEE Transactions on Pattern Analysis and Machine Intelligence 26 (2007)

6. Turk, M.A., Pentland, A.P.: Eigenfaces for recognition. Journal of Cognitive Neuroscience 3, 71-86 (1991)

7. Daugman, J.G.: High confidence visual recognition of persons by a test of statistical independence. IEEE Trans. Pattern Analysis and Machine Intelligence 15, 1148 $1161(1993)$

8. Jain, A., Nandakumarand, K., Ross, A.: Score normalization in multimodal biometric systems. Pattern recognition 2005 (to appear)

9. Snelick, R., Uludag, U., Mink, A., Indovina, M., Jain, A.: Large scale evaluation of multimodal biometric authentication using state-of-the-art systems (2005)

10. Li, S.Z., Chu, R.F., Ao, M., Zhang, L., He, R.: Highly accurate and fast face recognition using near infrared images. In: Proceedings of IAPR International Conference on Biometric (ICB-2006), Hong Kong, pp. 151-158 (2006) 